\title{
Asociación de síntomas emocionales e impulsividad con la compra compulsiva en universitarios $^{6}$
}

Emily González López

Psicóloga

Universidad EAFIT, Medellín, Colombia ygonzal2@eafit.edu.co

\section{Mariantonia Lemos}

Doctora en Psicología Universidad EAFIT, Medellín, Colombia Correo electrónico: mlemosh@eafit.edu.co
Recibido: 07/03/2018

Evaluado: 19/09/2018

Aceptado: 15/02/2019

\section{Resumen}

El objetivo de este estudio fue evaluar la presencia de compra compulsiva y su asociación con síntomas emocionales e impulsividad en estudiantes universitarios de la ciudad de Medellín (Colombia), así como llevar a cabo la adaptación y validación de la escala de adicción a las compras de Bergen (BSAS). Se evaluó a una muestra intencional de 98 estudiantes (68\% mujeres, edad media 20.4 años, D.T. $=2.19$ ) de cuatro universidades privadas. Los participantes completaron la BSAS, los inventarios de depresión y ansiedad de Beck, y la escala de impulsividad de Barrat. EL BSAS fue traducido al español y su estructura factorial se mantuvo, explicando el $47.4 \%$ de la varianza. El $23.5 \%$ de los evaluados presentó niveles significativos de compra compulsiva, $37.8 \%$ de ansiedad y $18.4 \%$ de depresión. Se encontraron correlaciones entre la compra compulsiva y ansiedad, depresión e impulsividad. Los resultados de la regresión lineal múltiple mostraron que la compra compulsiva se relaciona directamente con ansiedad e impulsividad, al controlar por sexo y edad.

Palabras clave

Compra compulsiva, adicción comportamental, ansiedad, depresión, impulsividad, análisis factorial.

6 Para citar este artículo: González, E. \& Lemos, M. (2020). Asociación de síntomas emocionales e impulsividad con la compra compulsiva en universitarios. Informes Psicológicos, 20(1), pp. 75-90http://dx.doi.org/10.18566/infpsic. v20n1a06 


\title{
Association of emotional symptoms and impulsivity with compulsive shopping in university students
}

\begin{abstract}
The objective of this study was to evaluate the presence of compulsive shopping and its association with emotional symptoms and impulsivity in university students of the city of Medellín (Colombia). An adaptation and a validation of the Bergen shopping addiction scale (BSAS) were applied. An intentional sample of 98 students ( $68 \%$ female, mean age 20.4 years, D.T. $=2.19$ ) from four private universities was evaluated. The participants completed the BSAS, Beck's depression and anxiety inventories, and Barrat's impulsivity scale. The BSAS was translated into Spanish and its factor structure was maintained, explaining $47.4 \%$ of the variance. $23.5 \%$ of those evaluated presented significant levels of compulsive purchase, $37.8 \%$ anxiety and $18.4 \%$ depression. Correlations were found between compulsive shopping and anxiety, depression and impulsivity. The results of the multiple linear regression showed that compulsive buying is directly related to anxiety and impulsivity, when controlling sex and age.
\end{abstract}

Keywords

Compulsive shopping, behavioral addiction, anxiety, depression, impulsivity, factor analysis.

\section{Associação dos sintomas emocionais e a impulsividade com a compra compulsiva em universitários}

Resumo

0 objetivo deste estudo foi avaliar a presença da compra compulsiva e sua associação com sintomas emocionais e a impulsividade em estudantes universitários da cidade de Medellín (Colômbia), bem como adaptar e validar a escala de dependência de compras de Bergen (BSAS). Foi avaliada uma amostra intencional de 98 estudantes (69\% mulheres, com idades ao redor de 20.4 anos, D.T. $=2.19)$ provenientes de quatro universidades privadas. 0s participantes completaram a BSAS, os inventários de depressão e ansiedade de Beck, e a escala de impulsividade de Barrat. 0 BSAS foi traduzido ao espanhol mantendo sua estrutura fatorial, explicando $047.4 \%$ da variância. 23,5\% dos avaliados apresentaram níveis significativos de compra compulsiva, $37,8 \%$ de ansiedade e $18,4 \%$ de depressão. Foram achadas correlações entre a compra compulsiva e a ansiedade, depressão e impulsividade. 0s resultados da regressão lineal múltipla mostraram que a compra compulsiva está diretamente relacionada à ansiedade $\mathrm{e}$ impulsividade, controlando por sexo e idade.

Palavras chave Compra compulsiva, dependência comportamental, ansiedade, depressão, impulsividade, análise fatorial. 


\section{ntroducción}

La compra compulsiva se entiende como el comportamiento de comprar que se da a partir de una motivación incontrolable por hacerlo, asociado a que se dedica mucho esfuerzo cognitivo alrededor de esta tarea (Andreassen, 2014). Este comportamiento de compra crónica y repetitiva parece presentarse como respuesta primaria a los acontecimientos o emociones negativas y se asocia con consecuencias nocivas y la afectación de la vida de quien lo parece (O'Guinn \& Faber, 1989). Actualmente es considerada por algunos como un tipo de adicción comportamental ya que se caracteriza por una necesidad, que está fuera del control voluntario, de continuar con la actividad, un incremento en la frecuencia y una dependencia psicológica a sus efectos placenteros aun cuando ésta tenga efectos negativos (Andreassen et al., 2013). Sin embargo, no es considerado un trastorno por la Asociación Americana de Psiquiatría, ya que no se tienen estudios suficientes para establecer criterios diagnósticos para este problema (Asociación Americana de Psiquiatría, 2014).

Son variadas las aproximaciones que tratan de explicar los factores que llevan a la compra compulsiva. Perspectivas sociales y culturales han hecho hincapié en que constituye un problema con raíces sociales importantes, en tanto valores capitalistas sobre el consumo y la adquisición, aspectos de la publicidad centrados en igualar la felicidad con la adquisición de objetos, llevan a que las personas presenten unos mayores niveles de consumo (Black, 2010; Grant, Potenza, Krishnan-Sarin, Cavallo \& Desai, 2011). Esto permitiría explicar por qué el fenómeno es más común en países con altos niveles de consumo (Thomas, Al-Menhali \& Humeidan, 2016). Así mismo se ha resaltado la importancia del proceso de socialización temprana asociado con el estilo de crianza y los aprendizajes familiares que se desarrollan en casa y que pueden lugar a rasgos como el materialismo cuando se estudia desde un enfoque más individual (Unger, Lyu \& Zimbardo, 2018). Sin embargo, este último enfoque también ha identificado variables individuales que llevan a que ciertas personas sean más vulnerables que otras frente al consumo y que pueden llegar a presentar patrones de compra de forma compulsiva (Shoham, Gavish \& Segev, 2014).

Al respecto, algunos estudios han encontrado que la compra compulsiva posee comorbilidad con los trastornos de ansiedad y depresión (Aboujaoude, 2014; Grant et al., 2011; Kim et al., 2018, OteroLópez \& Villardefrancos, 2014; Sohn \& Choi, 2014; Thomas et al., 2016; Zhang, Brook, Leukefeld \& Brook, 2016). Al parecer, las personas toman gran parte de sus decisiones de compra cuando se sienten ansiosos, ya que el comportamiento de compra reduce los sentimientos ansiógenos por medio de la recompensa y, en consecuencia, podría ocasionar que estos sujetos busquen repetir estos comportamientos con una mayor frecuencia debido a la conciencia que tienen de que la ansiedad va a disminuir con la conducta, empero, al no poder efectuarla de nuevo y no volver al escenario de compra, se pueden generar nuevamente sentimientos de ansiedad aún más intensos (Davenport, Houston \& Griffiths, 2012).

Por otra parte, la depresión es una de las variables altamente estudiadas en la compra compulsiva, ya que se considera 
que los sujetos con sentimientos negativos o con problemas múltiples podrían realizar transacciones comerciales con el fin de compensar esta situación de malestar que puede estar relacionada con ellos mismos y con su entorno familiar y social (Aravena, Herrera, Poblete \& Vera, 2006). Adicionalmente se ha encontrado que la compra compulsiva podría llevar a que las personas presenten síntomas depresivos. Un estudio llevado a cabo en los Emiratos Arabes encontró que los comportamientos de compra compulsiva explicaban el $42 \%$ de la varianza de síntomas depresivos en adultos que fueron evaluados en un centro comercial (BaniRshaid \& Alghraibeh, 2017).

Así mismo, una de las principales características de la compra compulsiva y en general para el desarrollo de la conducta adictiva, es la impulsividad (Aravena et al., 2006; Davenport et al., 2012; Grant et al., 2011), pues se considera que este problema para controlar los impulsos lleva al sujeto a experimentar un aumento de tensión antes de llevar a cabo una acción, al igual que la sensación de placer y gratificación posterior, razones por las cuales se menciona la relación de este rasgo con las adicciones comportamentales (Black, 2010; De Sola-Gutiérrez, RubioValladolid \& Rodríguez de Fonseca, 2013; Echeburúa \& De Corral, 1994; Hollander \& Allen, 2007; Williams \& Grisham, 2012). Al respecto, Black (2010) afirma que el $21 \%$ de los compradores compulsivos padecen un trastorno del control de los impulsos, mientras que sólo lo presentan un $4.2 \%$ de los compradores normales.

Con respecto a la evaluación y medición que se hace sobre este fenómeno, se identifican modelos como el modelo de formación y difusión del impulso de consumo (CIFE por sus siglas en inglés) en el que se reconoce la importancia de variables externas como el mercadeo, de rasgos de personalidad como la impulsividad, pero también del proceso de evaluación cognitiva, auto-control y su afectación por la emoción. Cabe anotar que este tipo de modelos exige la evaluación de variables mediadoras que requieren de aproximaciones experimentales (Dholakia, 2000; Baumeister, 2002). Así mismo existen estudios de enfoque mixto que buscan explorar la importancia de las creencias normativas sobre el consumo y su influencia sobre la impulsividad que lleva a la compra (Hausman, 2000; Unger et al., 2018). Estudios como el de Hausman (2000) evidencian que el hedonismo asociado a los productos que podrían obtenerse es una variable mediadora importante en la compra; así mismo el estudio de Unger et al. (2018) señala como las creencias asociadas con el tiempo, es decir, si se percibe que el futuro será mejor, el presente se ve de forma fatalista o el pasado fue peor podrían explicar diferencias en la compra compulsiva, explicando las diferencias en la frecuencia de compra compulsiva entre diferentes culturas.

Vale la pena mencionar que, aunque estas perspectivas son adecuadas para comprender el fenómeno de la compra compulsiva y poder establecer una mayor comprensión de las variables involucradas en el procesamiento de la información que lleva al comportamiento, es también necesario contar con instrumentos que evalúen la presencia o no de la compra compulsiva desde el ámbito clínico. Esto debido a que permite identificar aquellos que presentan de forma significativa el fenómeno y quienes están en riesgo de presentarlo, para poder establecer planes de acción desde la psicología clínica y de 
la salud, que les permita volver a adquirir un mayor autocontrol de su comportamiento y evitar problemas económicos y sociales que se relacionan con esta adicción (Zhang, Brook, Leukefeld, De La Rosa \& Brook, 2017).

Desde esta perspectiva de la evaluación del comportamiento y la identificación del comportamiento que podría llamarse anómalo es que se ha identificado que alrededor de una de cada veinte personas podrían llegar a presentar compra compulsiva en algún momento de sus vidas. Así mismo se ha afirmado que las personas jóvenes y las mujeres serán quienes estarían en mayor riesgo (Maraz, Griffiths \& Demetrovics, 2016; Villardefrancos \& Otero-López, 2017).

Dentro de esta perspectiva han surgido instrumentos enfocados en varios comportamientos adictivos, entre ellos la compra compulsiva (Christo et al., 2003; Faber \& O'Guinn, 1992), sin embargo, la escala de adicción a las compras de Bergen (BSAS por sus siglas en inglés), es una escala de sólo siete ítems que se centra en evaluar los componentes básicos de las adicciones (prominencia, modificación del estado de ánimo, conflicto, tolerancia, recaída, retirada y los problemas asociados a la conducta) en el ámbito de la compra compulsiva (Andreassen et al., 2015). Vale la pena anotar que posterior a esta escala se han desarrollado instrumentos que la toman como base para la evaluación de este comportamiento, por ejemplo, en las compras online (Manchiraju, Sadachar \& Ridgway, 2017); no obstante, ha sido reconocido por los mismos autores de la escala que la escala BSAS sería adecuada para evaluar este comportamiento en ámbitos diversos, tales como mediante el Internet (Griffiths et al., 2016).

Con respecto a los estudios en Colombia, se pudo observar que se han centrado en la relación de la compra compulsiva con la discrepancia del ser (Botero, Gutiérrez, Manjarrés \& Torres, 2008) y el materialismo (Luna-Arocas, Puello-Arrieta, \& Botero, 2004); así como la descripción del comportamiento en grupos poblacionales específicos, p.e. homosexuales (Giraldo-Oliveros \& Alvarado-López, 2006); sin embargo, estas investigaciones han utilizado escalas diversas para la identificación de quienes presentan compra compulsiva, sin que ninguna de ellas esté centrada realmente en este cuadro, ya que al momento no se cuenta en el país con una escalas validada para evaluar la compra compulsiva,

De manera complementaria, autores como Hollander y Allen (2007) han señalado la importancia de realizar estudios sobre la prevalencia de la compra compulsiva, sus factores de vulnerabilidad y los mecanismos que la subyacen, buscando comprenderla mejor, de tal manera que se facilite su conocimiento y puedan desarrollarse acciones terapéuticas eficaces para las personas que la padecen. Lo anterior llevó a pensar en la necesidad de llevar a cabo este estudio con dos objetivos: 1. Estudiar la prevalencia de este fenómeno en universitarios, los cuales según lo reportado previamente hacen parte de la población en mayor riesgo, y su asociación con ansiedad, depresión e impulsividad, como factores de vulnerabilidad frente a la misma. 2. Realizar el proceso de traducción, adaptación y validación estructural de la escala de adicción a las compras de Bergen, construida con 
el fin de identificar personas con compra compulsiva (Andreassen et al., 2015).

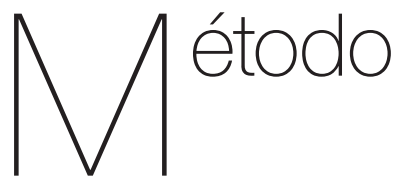

\section{Diseño de estudio}

Investigación cuantitativa, descriptivo correlacional de corte transversal. Es decir, se trató de un estudio que buscaba especificar la frecuencia de las variables medidas y establecer relaciones entre ellas (Hernández, Fernández \& Baptista, 2010).

\section{Población y muestra}

La población de referencia estuvo constituida por estudiantes de universidades privadas de la ciudad de Medellín (Colombia). Para este estudio se llevó a cabo un muestreo intencional con 98 adultos jóvenes. La muestra estuvo conformada por estudiantes con una edad promedio de 20.4 años (D.T. = 2.19), con un rango de edad entre los 18 y 29 años. De ésta hicieron parte 67 mujeres (68\%) y 31 hombres (32\%) (Tabla 1).

Tabla 1.

Variables demográficas de la muestra universitaria evaluada

\begin{tabular}{lccccc}
\hline Datos Demográficos & N & \% & M & DT \\
\hline \multirow{2}{*}{ Sexo } & Mujer & 67 & 68 & & \\
& Hombre & 31 & 32 & & \\
\hline \multirow{2}{*}{ Edad } & 18 a 22 & 83 & 85 & 19.5 & 1.41 \\
& 23 a 26 & 13 & 13 & 23.5 & .52 \\
& 27 a 29 & 2 & 2 & 28.0 & 1.41
\end{tabular}

Nota: n: número de personas, M: Media, DT: desviación típica Fuente: elaboración propia.

\section{Instrumentos}

Escala de adicción a las compras de Bergen (BSAS por sus siglas en inglés; Andreassen et al., 2015). Escala de siete reactivos, evalúa los componentes básicos de las adicciones (prominencia, modificación del estado de ánimo, conflicto, tolerancia, recaída, retirada y los problemas asociados a la conducta) creada por Andreassen et al. (2015). Cada reactivo se debe responder de acuerdo con una escala Likert que va de 0 "completamente en desacuerdo" y 4 "completamente de acuerdo". La versión en inglés presenta un alfa de Cronbach de .867. Para el presente estudio se llevó a cabo la traducción y retraducción por dos personas bilingües independientes en cada fase (cuatro traductores en total), para obtener una versión en español que fuera consistente con la versión original la cual fue revisada y aprobada por uno de los autores de la prueba (M.D. Griffiths). La confiabilidad del instrumento en este estudio fue de .85 .

Escala de impulsividad de Barratt (BIS15S, Orozco-Cabal, Rodríguez, Herin, Gempeler, \& Uribe, 2010): validación colombiana de la escala de Barratt (BIS-15), la cual es la versión corta de la escala original, la cual contenía 30 ítems y fue creada hace más de 50 años para medir impulsividad (Patton \& Stanford, 1995). Esta versión fue desarrollada por Spinella en 2007 utilizando una muestra de 700 sujetos, donde los 30 ítems de la versión original se sometieron a un análisis de componentes principales y se seleccionaron los cinco reactivos de cada factor que presentaban las cargas más elevadas, obteniendo así una consistencia total de .81. Se trata de un test de auto-reporte que consta de 15 ítems agrupados en 
tres subescalas: Impulsividad cognitiva, motora y no planeada. Cada reactivo se responde de acuerdo con una escala Likert que va de 1 (raramente o nunca) a 4 (siempre o casi siempre). Posteriormente, Orozco-Cabal et al. (2010) validaron esta versión resumida en población colombiana, utilizando una muestra de 447 sujetos arrojando una consistencia interna de .79 y una confiabilidad test-retest de .80 . Para este estudio, el instrumento tuvo una confiabilidad general de .52, y de .71, .77 para las subescalas de Impulsividad Motora e Impulsividad Atencional, respectivamente. La subescala de Impulsividad Atencional no presentó una confiabilidad adecuada $(\alpha=.20)$.

Inventario de ansiedad de Beck (BAI por sus siglas en inglés; Beck \& Steer, 2011). Cuestionario de 21 ítems que mide la gravedad de la ansiedad en adultos y adolescentes. Su tiempo de aplicación es de 5 a 10 minutos. En la validación original, el BAl arrojó un nivel de consistencia interna alto (.92). La prueba test-retest con una diferencia de una semana tuvo una correlación de .75. Con respecto a la validez concurrente y divergente se encontró que el BAI correlacionó con el HAM-A en .51, HAM-D .25, BDI .48, STAI rasgo .58 y STAl estado .47 (Beck \& Steer, 2011). En la validación española, Fernández y Navarro (2003) concluyen que el BAl cubre 13 de los 29 síntomas distintos que, según el DSM IV, definen los trastornos de ansiedad. Los índices de consistencia interna fluctuaron entre .85 en una muestra de adultos general y .93 en la otra. En el presente estudio el BAI presentó una consistencia interna de .91.

Inventario de depresión de Beck, segunda versión (BDI II por sus siglas en inglés, Beck, Steer \& Brown, 2011).
Cuestionario de autoaplicación compuesto por 21 ítems. Ha sido diseñado para evaluar la gravedad de sintomatología depresiva en adultos y adolescentes con una edad mínima de 13 años. Este cuestionario se desarrolló con base en la versión original (Beck, Steer \& Brown, 1996; Beck, Ward, Mendelson, Mock, \& Erbaugh, 1961) para la evaluación de los síntomas que se corresponden con los criterios diagnósticos de los trastornos depresivos recogidos en el DSM IV y que están vigentes en el DSM 5. Su tiempo de aplicación es de 5 a 10 minutos. La validación original y española del instrumento mostraron índices de confiabilidad apropiados (.92 para la muestra americana y entre .87 y .91 para las muestras españolas). Adicionalmente, los análisis factoriales en ambas validaciones mostraron la existencia de dos factores principales que explicaban la mayor proporción de la varianza y se relacionan con la sintomatología somática y cognitiva de la depresión (Beck et al., 2011; Sanz, Perdigón, \& Vásquez, 2003). En este estudio el instrumento presentó una consistencia interna de .88.

\section{Procedimiento}

Los participantes fueron invitados a hacer parte de este estudio mediante contacto directo en cuatro universidades privadas de Medellín, Colombia. Se buscó que la cuota muestral de las universidades fuera semejante. Los estudiantes que aceptaron participar posterior a la explicación sobre el objetivo del estudio y el procedimiento, firmaron el consentimiento informado y les fue entregado un sobre con el protocolo de evaluación constituído por las pruebas anteriormente mencionadas. 


\section{Análisis estadístico}

Las respuestas de los participantes fueron digitadas en una base de datos en Excel, la cual fue importada al SPSS versión 25 para su análisis. Inicialmente se obtuvieron las puntuaciones subtotales y totales por escala y los índices de confiabilidad de los instrumentos. Previo al uso de la Escala de adicción a las compras de Bergen fue necesario llevar a cabo un análisis factorial exploratorio para verificar su estructura factorial. Para este análisis se utilizó el método de componentes principales, el cual tiene como objetivo resumir la mayor parte de la información contenida en los reactivos en un número menor de variables o componentes (Mulaik, 2009). Para evaluar la conveniencia de este análisis, se utilizaron la medida de suficiencia del muestreo (MSA) y la prueba de esfericidad de Bartlett. Si MSA obtiene un valor superior a .80 y la prueba de Barlett es estadísticamente significativa, se puede determinar la conveniencia del análisis. Para evaluar el número de factores, se utilizó el criterio de raíz latente. Según este criterio, el número de factores a retener depende del número de factores que obtengan autovalores mayores a 1. En cuanto a la carga factorial, en muestras de 100 observaciones valores de .55 o más se consideran significativos (Hair, Anderson, Tatham, \& Black, 2008).

Posteriormente, se analizó la prevalencia de compra compulsiva y se realizó un análisis de correlaciones para verificar la asociación de la compra compulsiva con las demás variables evaluadas en este estudio (ansiedad, depresión e impulsividad). Finalmente, se estableció un modelo de regresión lineal múltiple controlando por el sexo y la edad, de tal forma que se pudieran observar las asociaciones entre las variables de forma conjunta. Para evaluar si existía multicolinealidad en las variables dependientes, se tomaron como criterios el estadístico de tolerancia y el factor de inflación de la varianza (VIF). Según Meyers, Gamst y Guarino (2006), un valor de .1 o menos en tolerancia o un valor de 10 o más en el VIF sería indicador de multicolinealidad. De forma similar el análisis de los residuos implicó estimar el estadístico de Durbin Watson y el análisis de la gráfica de los residuos estandarizados contra los valores predichos estandarizados.

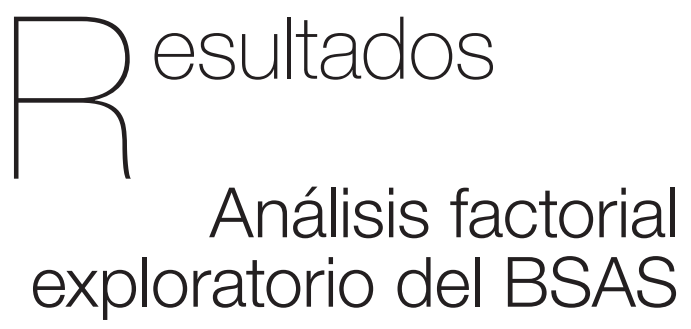

Las pruebas MSA y de esfericidad de Bartlett indican que el análisis de componentes principales es adecuado $(\mathrm{MSA}=.82 ; \chi 2(21, \mathrm{~N}=98)=287.53, \mathrm{p}=$ .00). El primer factor componente obtuvo un autovalor igual a 3.81, mientras que el valor de autovaloraciones para el segundo componente fue .87. Este resultado indica que la información de los reactivos puede resumirse en un sólo componente, el cual explica $47.40 \%$ de la varianza de los ítems. Como puede observarse, dado que teníamos una muestra de 98 y que los valores de las cargas factoriales fueron superiores a .55 , puede afirmarse que todas resultaron significativas (ver Tabla 2). El modelo se corresponde con el modelo original propuesto por Andreassen et al. (2015). 
Tabla 2.

Cargas factoriales de la escala de adicción a las compras de Bergen

\begin{tabular}{lccc}
\hline Factor & Items & $\begin{array}{c}\text { Carga } \\
\text { factorial }\end{array}$ & $\begin{array}{c}\text { Varianza } \\
\text { explicada }\end{array}$ \\
\hline & 1 & .76 & $47.40 \%$ \\
& 2 & .63 & \\
& 3 & .76 & \\
Adicción a las & 4 & .86 & \\
compras & 5 & .73 & \\
& 6 & .66 & \\
& 7 & .75 & \\
\hline
\end{tabular}

Fuente: elaboración propia

\section{Prevalencias de Compra compulsiva, depresión y ansiedad}

Se encontró que el $23.5 \%$ de la muestra presentaba niveles de compra compulsiva significativos ( $n=23$ ), el $37.8 \%$ presentó ansiedad $(n=37)$ y el $18.4 \%$ depresión ( $n=18)$. Así mismo, se evidenció una asociación entre la compra compulsiva y el sexo, siendo ésta más frecuente en las mujeres, Fisher $\mathrm{X}^{2}(1)=4.802, \mathrm{p}<$ .05 (un $9.7 \%$ de los hombres $(n=3)$ frente a un $29.9 \%$ de las mujeres $(n=20)$.

\section{Relación entre la compra compulsiva con los niveles de depresión, ansiedad e impulsividad}

Al realizar las correlaciones entre las variables en estudio se evidenció que la compra compulsiva se encuentra positivamente asociada con la ansiedad ( $p<.001$ ), la impulsividad ( $p<.05)$, y la depresión $(p<$.05) (Tabla 3).

Tabla 3.

Correlaciones entre BSAS y las puntuaciones de Ansiedad, Depresión e Impulsividad, con una muestra de población universitaria $(n=98)$.

\begin{tabular}{lccc}
\hline & $\begin{array}{c}\text { Compra } \\
\text { Compulsiva }\end{array}$ & Impulsividad & Ansiedad \\
\hline Impulsividad & $.21^{*}$ & & \\
Ansiedad & $.43^{\star *}$ & $.22^{*}$ & \\
Depresión & $.22^{*}$ & $.24^{*}$ & $.50^{* *}$ \\
\hline
\end{tabular}

Nota: ${ }^{*} p<.05,{ }^{* *} p<.001$; Fuente: elaboración propia

Finalmente, se llevó a cabo una regresión lineal por pasos para establecer aquellas variables que podrían relacionarse con la aparición de compra compulsiva, controlando por la edad y del sexo. Se encontró que existe una relación lineal entre la compra compulsiva con las variables del estudio, $F(5,97)=8.914, p$ $<.001$, las cuales explicaron el 29\% de la varianza de la compra compulsiva. Al respecto del modelo, se evidenció que ser mujer $(p<.01)$, presentar síntomas de ansiedad $(p<.001)$ y rasgos de impulsividad $(p<.01)$ eran factores asociados con la compra compulsiva (Tabla 4).

Cabe anotar que todas las variables dependientes presentaron valores de tolerancia mayores de .69 y el VIF que presentó un mayor valor fue el de Ansiedad con 1.475. El valor de Durbin Watson fue de 1.606 y la gráfica de los residuos estandarizados contra los valores predichos estandarizados no evidenció problemas de heteroscedasticidad y no linealidad. 
Tabla 4.

Regresión lineal de variables asociadas con la compra compulsiva.

\begin{tabular}{lccccc}
\hline & \multicolumn{1}{c}{ B } & $\begin{array}{c}\text { Error } \\
\text { estándar }\end{array}$ & Beta & T & Sig \\
\hline \multicolumn{2}{l}{ Paso 1 $(\mathrm{R} 2=0.058)$} & & & & \\
Edad & .292 & .270 & .113 & 1.083 & .281 \\
Sexo & -3.547 & 1.263 & -.292 & -2.809 & .006 \\
Paso 2 (R2 = 0.290) & & & & \\
Impulsividad & .478 & .168 & .266 & 2.853 & .005 \\
Ansiedad & .192 & .052 & .382 & 3.673 & .000 \\
Depresión & -.028 & .069 & -.042 & -.413 & .680 \\
\hline
\end{tabular}

Nota: Variable dependiente: BSAS ( $N=98)$. Fuente: elaboración propia.

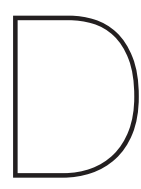

\section{iscusión}

Esta investigación buscó relacionar la sintomatología depresiva, ansiosa y rasgos de impulsividad con la compra compulsiva, los cuales podrían considerarse factores de riesgo o vulnerabilidad en los estudiantes universitarios para que lleguen a desarrollar este problema, que si bien no está especificado en el DSM-5, algunos estudios indican que se trata de un problema de control de impulsos con comorbilidad con trastornos clínicos como la ansiedad y la depresión (Black, 2010). Se encontró que la compra compulsiva se relaciona con niveles de ansiedad e impulsividad, pero no con sintomatología depresiva. Así mismo, este estudio se propuso llevar a cabo la validación de la escala de adicción a las compras de Bergen, construida con el fin de identificar personas con compra compulsiva. Nuestros resultados señalan que se trata de una escala con estructura factorial y coeficientes de confiabilidad adecuados para su uso.

Estudios previos han señalado que la ansiedad es uno de los mayores factores de riesgo para las adicciones patológicas, tal como la compra compulsiva (Aravena et al., 2006). Al respecto, la ansiedad aparecería previo al acto de comprar, disminuyendo posterior al mismo; pero también ésta estaría presente, de manera general, debido a la culpa y las deudas originadas por las compras; así como en los momentos que la actividad de comprar no puede realizarse (Davenport et al., 2012; De Sola-Gutiérrez et al., 2013; Echeburúa \& De Corral, 1994; Grant et al., 2011; Sohn \& Choi, 2014).

Por otra parte, se ha afirmado que la impulsividad es una de las características principales de la compra compulsiva, y en general para el desarrollo de la conducta adictiva (Dholakia, 2000; Hausman, 2000; Kim et al., 2018). Así, los resultados de este estudio son consistentes con lo afirmado por Dholakia (2000) en el modelo CIFE acerca de la importancia del impulso de consumo en la compra compulsiva. Según lo expuesto por este autor la impulsividad por si sola podría ser tan efectiva para producir la experiencia de consumo, mientras que en personas con una presencia menor del rasgo la interacción de éste con los estímulos del mercadeo podría llevar a la misma acción. Cabe anotar que, aunque nuestro estudio no evalúa las variables ambientales relacionadas con el marcadeo, sí señala la importancia de la impulsividad como rasgo en esta conducta. Adicionalmente estos resultados señalan que las personas con un rasgo impulsivo tienen la tendencia a reaccionar frente al estrés y la ansiedad realizando acciones que les generen placer inmediato, pues hay una urgencia motivada por la situación de tensión que lleva a la acción, que finalmente relaja al sujeto momentáneamente, e inicia el ciclo nuevamente (De Sola-Gutiérrez et al., 2013; Davenport et al., 2012; Andreassen 
et al., 2013). No obstante, dentro del modelo CIFE se reconoce que una emoción positiva también podría favorecer la evaluación cognitiva que dispararía el consumo del individuo.

Frente a la depresión, no se encontró que ésta se asociara con la compra compulsiva. Esto difiere de otros estudios realizados en este campo los cuales afirman que las personas con esta condición podrían realizar transacciones comerciales con el fin de compensar una situación de malestar que puede originarse en síntomas depresivos (Aravena et al., 2006; Black, 2010; De Sola-Gutiérrez et al., 2013; Echeburúa \& De Corral, 1994; Hollander \& Allen, 2007; Koran, Faber, Aboujaoude, Large \& Serpe, 2007; Sohn \& Choi, 2014; Thomas et al., 2016). No obstante, es importante aclarar que en este estudio la depresión por sí sola presentó correlación significativa con la aparición de la compra compulsiva, pero al controlarse por las demás variables esta relación pierde significancia, lo que llevaría a pensar que dicha relación podría estar mediada por la ansiedad, tal y como fue reportado en el estudio de Williams y Grisham (2012) en el cual se encontró que la depresión estaba asociada con la compra compulsiva, pero el estrés y la ansiedad sí.

Por otra parte, la prevalencia encontrada de compra compulsiva en la muestra evaluada fue mucho mayor a la reportada en otros estudios (Koran et al., 2007; Maraz et al., 2016), aunque cercana a la reportada en estudiantes universitarios españoles, donde se encontró una prevalencia del 16\% (Ruiz-Olivares et al., 2010) y menor que la que fue reportada en los Emiratos Arábes, en la que se reportó una proporción del $40 \%$ de la población evaluada (Thomas et al., 2016). Estas diferencias podrían deberse a razones culturales, evidenciando la tendencia cultural al consumo que se ha venido dando en Colombia.

Vale la pena señalar que la prevalencia de ansiedad es semejante a la que se ha reportado en otros estudios con población universitaria (Medrano \& Moretti, 2013); sin embargo la prevalencia de depresión fue más alta que la que se ha reportado en otros estudios con población universitaria (Cabas, González \& Mendoza, 2017), pero menor a la encontrada en otros estudios en universidades colombianas, donde la proporción alcanzó el 36.3\% (Jaramillo-Toro et al., 2018; Pabón, Espinosa, Correa, Ríos \& Gutiérrez, 2018). Esto podría tener relación con las mayores cifras frente a la compra compulsiva, al comprender que ésta tiende a configurarse como una estrategia de afrontamiento ante el malestar emocional.

Este estudio también confirmó la asociación entre la compra compulsiva y el ser mujer, el cual había sido reportado en otras investigaciones (De Sola-Gutiérrez et al., 2013). Esta relación podría explicarse por mayores de depresión y ansiedad en mujeres (Asociación Americana de Psiquiatría, 2014; Mazo, Londoño \& Gutiérrez, 2013). Una posible hipótesis de la diferencia entre sexos podría arrojarla un estudio llevado a cabo en universitarios canadienses que encontró que la ansiedad está asociada con mayores probabilidades de presentar compra compulsiva, mientras que la depresión y la culpa aparecen de forma posterior. Con respecto a los sexos fueron las mujeres quienes reportaron que presentaban mayor comportamiento de compra compulsiva cuando se sentían ansiosos y culpa posterior a ésta. Los hombres eran más proclives 
a reportar sentimientos negativos frente a la compra (Gallagher, Watt, Weaver \& Murphy, 2017).

Cabe anotar que, a diferencia de estudios previos, no se encontró una relación entre la edad y la compra compulsiva, donde a mayor edad menor riesgo (RuizOlivares, Lucena, Pino \& Herruzo, 2010). Es posible que este resultado pueda deberse a que sólo se evaluó un rango de edad, así como por el hecho que se trató de una muestra no representativa sino intencional, lo que pudo haber sesgado este resultado.

Con respecto al objetivo secundario, este estudio tradujo y realizó un análisis factorial exploratorio de la escala de adicción a las compras de Bergen, herramienta que puede ser utilizada en investigaciones futuras del tema de compra compulsiva, aun de forma electrónica. Al respecto, este estudio encontró que todos los ítems de la prueba presentaron cargas factoriales adecuadas y se agrupan en un solo factor, tal y como fue descrito por Andreassen et al. (2015) en el estudio original. Al ser ésta una escala de sólo siete ítems, podría convertirse en un instrumento de fácil utilización por la comunidad interesada en esta temática.

Este estudio presenta limitaciones que deben ser señaladas. Al ser un estudio transversal, los hallazgos acerca de las relaciones entre variables no pueden entenderse como factores de riesgo específicamente, sino como asociaciones; sin embargo, se hacen afirmaciones de posible vulnerabilidad basados en la evidencia científica disponible. En segundo lugar, la muestra de este estudio fue intencional, de tal forma que puede haber limitaciones para la generalización de los resultados a la población universitaria en general. Adicionalmente vale la pena señalar que estos resultados arrojan evidencia de síntomas emocionales y el rasgo de impulsividad dentro de la compra compulsiva; sin embargo, este fenómeno debe entenderse teniendo en cuenta la importancia de variables ambientales como el mercadeo y los valores culturales asociados al consumo.

En síntesis, los resultados arrojados por esta investigación permiten confirmar la relación existente entre la compra compulsiva, la impulsividad y la ansiedad. Estos datos pueden servir de insumo para la realización de programas orientados a la prevención de la compra compulsiva en estudiantes universitarios en los cuales la prevalencia de ansiedad e impulsividad es importante.

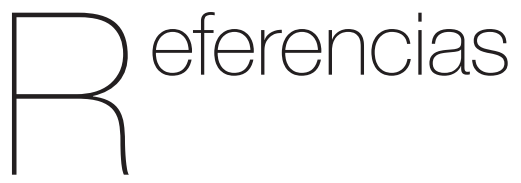

Aboujaoude, E. (2014). Compulsive buying disorder: a review and update. Current Pharmaceutical Design, 20(25), 4021 4025. https://doi.org/10.2174/13816128113 199990618

Andreassen, C. S. (2014). Shopping addiction: an overview. Journal of Norwegian Psychological Association, 51, 194-209.

Andreassen, C. S., Griffiths, M. D., Gjertsen, S. R., Krossbakken, E., Kvam, S. \& Pallesen, S. (2013). The relationships between behavioral addictions and the five-factor model of personality. Journal of Behavioral Addictions, 2(2), 90-99. https://doi. org/10.1556/JBA.2.2013.003 
Andreassen, C.S., Griffiths, M. D., Pallesen, S., Bilder, R.M., Torsheim, T. \& Aboujaoude, E. (2015). The Bergen Shopping Addiction Scale: reliability and validity of a brief screening test. Frontiers in Psychology, 6, 1-11. https://doi.org/10.3389/ fpsyg.2015.01374

Aravena, V., Herrera, V., Poblete, P., \& Vera, D. (2006). Consumo Patológico: Compra Impulsiva y Compulsiva. Investigación en psicología del consumidor. Recuperado de http://www.inpsicon.com/ estudios_realizados/espanol/Aravena_ Esp_04052007.pdf

Asociación Americana de Psiquiatría (2014). Manual diagnóstico y estadístico de los trastornos mentales (DSM-5). $5^{\mathrm{a}}$ Ed. Arlington, VA: Asociación Americana de Psiquiatría

Baumeister, R. F. (2002). Yielding to temptation: Self-control failure, impulsive purchasing, and consumer behavior. Journal of Consumer Research, 28(4), 670-676. https://doi.org/10.1086/338209

Bani-Rshaid, A. M., \& Alghraibeh, A. M. (2017). Relationship between compulsive buying and depressive symptoms among males and females. Journal of ObsessiveCompulsive and Related Disorders, 14, 47-50. $\quad$ https://doi.org/10.1016/j. jocrd.2017.05.004

Beck, A. T., \& Steer, R. A. (2011). Inventario de Ansiedad de Beck - Manual (J. Sanz, Trans.). Madrid: Pearson Clinical \& Talent Assessment España.

Beck, A. T., Steer, R. A., \& Brown, G. K. (1996). Manual for the Beck Depression InventoryII (2nd ed.). San Antonio: Harcourt Brace \& Company.
Beck, A. T., Steer, R. A., \& Brown, G. K. (2011). Inventario de Depresión de Beck II- Manual (J. Sanz, Trans.). Madrid: Pearson Clinical \& Talent Assessment España.

Beck, A. T., Ward, C. H., Mendelson, M., Mock, J., \& Erbaugh, J. K. (1961). An inventory for measuring depression. Archives of General Psychiatry, 4(6), 561-571. https://doi.org/10.1001/ archpsyc.1961.01710120031004

Black, D. W. (2010). Compra compulsiva: una revisión. Revista de Toxicomanía y Salud Mental, 28(3), 61-413.

Botero, M. M., Gutiérrez, M., Manjarrés, L. K., \& Torres, T. (2008). La Relación del SELF con el consumo en hombres y mujeres entre 18 y 24 años, estudiantes de la Universidad del Norte. Psicología desde el Caribe, (21), 1-31.

Cabas, K., González, Y. \& Mendoza, C. (2018). Funcionamiento ejecutivo y depresión en universitarios con normopeso, sobrepeso y obesidad tipo I. Informes Psicológicos, 18(1), 133-144. http://dx.doi.org/10.18566/ infpsic.v18n1a07

Christo, G., Jones, S., Haylett, S., Stephenson, G., Lefever, R. M., \& Lefever, R. (2003). The shorter PROMIS questionnaire: further validation of a tool for simultaneous assessment of multiple addictive behaviors. Addictive Behaviors, 28, 225-248. https:// doi.org/10.1016/S0306-4603(01)00231-3

Davenport, K., Houston J. E, \& Griffiths, M. D. (2012). Excessive eating and compulsive buying behaviours in women: an empirical pilot study examining reward sensitivity, anxiety, impulsivity, self-esteem and social desirability. International Journal of Mental 
Health and Addiction, 10, 474-489. https:// doi.org/10.1007/s11469-011-9332-7

De Sola-Gutiérrez, J., Rubio-Valladolid, G, \& Rodríguez de Fonseca, F. (2013). La impulsividad: ¿antesala de las adicciones comportamentales? Health and Addictions, 13(2), 145-155. https://doi.org/10.21134/ haaj.v13i2.211

Dholakia, U. M. (2000). Temptation and resistance: An integrated model of consumption impulse formation and enactment. Psychology \& Marketing, 17(11), 955-982. https://doi.org/10.1002/15206793(200011)17:11<955:: AID MAR3>3.0.CO;2-J

Echeburúa, E., \& De Corral, P. (1994). Adicciones psicológicas: Más allá de la metáfora. Clínica y Salud, 5(3), 251-258

Faber, R. J., \& O'Guinn, T. C. (1992). A clinical screener for compulsive buying. Journal of Consumer Research, 19(3), 459-469. https://doi.org/10.1086/209315

Fernández, J. S., \& Navarro, M. E. (2003). Propiedades psicométricas de una versión española del Inventario de Ansiedad de Beck (BAl) en estudiantes universitarios. Ansiedad y Estrés, 9(1), 59-84.

Gallagher, C. E., Watt, M. C., Weaver, A. D., \& Murphy, K. A. (2017). "I fear, therefore, I shop!" exploring anxiety sensitivity in relation to compulsive buying. Personality and Individual Differences, 104, 37-42. https://doi.org/10.1016/j.paid.2016.07.023

Giraldo-Oliveros, M. E, \& Alvarado-López, V.G. (2006). Compra impulsiva en el homosexual masculino. Pensamiento \& Gestión, 20, 125-164.
Grant, J. E., Potenza, M. N., Krishnan-Sarin, S., Cavallo, D. A, \& Desai, R. A. (2011). Shopping Problems among High School Students. Comprehensive Psychiatry, 52(3), 247-252. https://doi.org/10.1016/j. comppsych.2010.06.006

Griffiths, M. D., Andreassen, C. S., Pallesen, S., Bilder, R. M., Torsheim, T., \& Aboujaoude, E. (2016). When is a new scale not a new scale? The case of the Bergen Shopping Addiction Scale and the Compulsive Online Shopping Scale. International Journal of Mental Health and Addiction, 14(6), 1107-1110. https://doi.org/10.1007/ s11469-016-9711-1

Hausman,A.(2000)Amulti-methodinvestigation of consumer motivations in impulse buying behaviour. Journal of Consumer Marketing, 17(5), 403-426, https://doi. org/10.1108/07363760010341045

Hair, J. F., Anderson, R. E., Tatham, R., \& Black, W. C. (2008). Análisis Multivariante. Madrid: Análisis Multivariante. Prentice Hall: Madrid

Hernández, R., Fernández, C. \& Baptista, M. P. (2010). Metodología de la investigación, $5^{a}$ ed. México D.F: McGraw- Hill.

Hollander, E., \& Allen, A. (2007). ¿Es la compra compulsiva un verdadero trastorno, y es compulsivo en realidad? American Journal of Psychiatry- edición española, 10(1), 4-6.

Jaramillo-Toro, C., Martínez, J. W., GómezGonzález, J. F., Mesa, P., Otálvaro, S., \& Sánchez-Duque, J. A. (2018). Sintomatología depresiva en una población universitaria de Colombia: Prevalencia, factores relacionados y validación de dos instrumentos para tamizaje. Revista Chilena de Neuro-psiquiatría, 56(1), 
18-27. http://dx.doi.org/10.4067/ s0717-92272018000100018

Kim, H. S., Hodgins, D. C., Torres, A. R., Fontenelle, L. F., do Rosário, M. C., de Mathis, M. A., ... \& Tavares, H. (2018). Dual diagnosis of obsessive compulsive and compulsive buying disorders: Demographic, clinical, and psychiatric correlates. Comprehensive Psychiatry, 86, 67-73. https://doi.org/10.1016/j. comppsych.2018.07.013

Koran, L. M., Faber, R. J., Aboujaoude, E., Large, M. D, \& Serpe, R.T. (2007). Estimación de la prevalencia del comportamiento de compra compulsiva en Estados Unidos. American Journal of Psychiatry- edición española, 10(1), 45-51.

Luna-Arocas, R., Puello-Arrieta, S, \& Botero, M. M. (2004). La compra impulsiva y el materialismo en los jóvenes: Estudio exploratorio en estudiantes universitarios de Barranquilla (Colombia). Psicología desde el Caribe, 14, 1-26.

Maraz, A., Griffiths, M. D., \& Demetrovics, Z. (2016). The prevalence of compulsive buying: a meta-analysis. Addiction, 111(3), 408-419. https://doi.org/10.1111/add.13223

Manchiraju, S., Sadachar, A., \& Ridgway, J. L. (2017). The compulsive online shopping scale (COSS): development and validation using panel data. International Journal of Mental Health and Addiction, 15(1), 209-223. https://doi.org/10.1007/ s11469-016-9662-6

Mazo, R., Londoño, K. \& Gutiérrez, Y.F. (2013). Niveles de estrés académico en estudiantes universitarios. Informes Psicológicos, 13(2), 121-134.
Medrano, L.A. \& Moretti, L. (2013). Eficacia de un programa de entrenamiento para disminuir manifestaciones cognitivas de ansiedad ante los exámenes en estudiantes universitarios. Informes Psicológicos, 13(1), 41-52.

Meyers, L.S., Gamst, G. \& Guarino, A.J. (2006). Applied Multivariate Research, Design and Interpretation. Thousand Oaks, California: Sage Publications.

Mulaik, S. A. (2018). Foundations of factor analysis, $2^{\text {nd }}$ ed. Boca Ratón FL: Taylor \& Francis

O'Guinn, T. C. \& Faber, R.J. (1989). Compulsive buying: A phenomenological exploration. Journal of Consumer Research, 16(2), 147157. https://doi.org/10.1086/209204

Orozco-Cabal, L., Rodríguez, M., Herin, D., Gempeler, J., \& Uribe, M. (2010). Validity and reliability of the abbreviated Barratt Impulsiveness Scale in Spanish (BIS-15S). Revista Colombiana de Psiquiatría, 39(1), 93-109. https://doi. org/10.1016/s0034-7450(14)60239-0

Otero-López, J. M., \& Villardefrancos, E. (2014). Prevalence, sociodemographic factors, psychological distress, and coping strategies related to compulsive buying: a cross sectional study in Galicia, Spain. BMC Psychiatry, 14, 1-12. https:// doi.org/10.1186/1471-244X-14-101

Pabón, J. B., Espinosa, J. F. S., Correa, Y. M., Ríos, D. A. V., \& Gutiérrez, U. R. (2018). Prevalencia de Sintomatología Depresiva en estudiantes del programa de Medicina de la Universidad de Caldas, Manizales-Colombia. Revista Médica de Risaralda, 24(1), 20-23. Recuperado de 
http://www.scielo.org.co/pdf/rmri/v24n1/ v24n1a04.pdf

Patton, J. H., \& Stanford, M. S. (1995). Factor structure of the Barratt impulsiveness scale. Journal of Clinical Psychology, 51(6), 768-774. https://doi.org/10.1016/j. paid.2009.04.008

Ruiz-Olivares, R., Lucena, V., Pino, M. J, \& Herruzo, J. (2010). Análisis de comportamientos relacionados con el uso/ abuso de Internet, teléfono móvil, compras y juego en estudiantes universitarios. Adicciones, 22(4), 301-310. https://doi. org/10.20882/adicciones.171

Sanz, J., Perdigón, A. L. \& Vásquez C. (2003). Adaptación española del Inventario para la Depresión de Beck-II (BDI-II): 2. Propiedades psicométricas en población general. Clínica y salud 14(3), 249-280.

Shoham, A., Gavish, Y., \& Segev, S. (2014). A Cross-Cultural Analysis of Impulsive and Compulsive Buying Behaviors among Israeli and U.S. Consumers: The Influence of Personal Traits and Cultural Values. Journal of International Consumer Marketing 27, 187-206. https://doi.org/10.10 80/08961530.2014.1000507

Sohn, S. H, \& Choi, Y. J. (2014). Phases of Shopping Addiction Evidenced by Experiences of Compulsive Buyers. International Journal of Mental Health and Addiction, 12(3), 243-254. https://doi. org/10.1007/s11469-013-9449-y

Spinella, M. (2007). Normative data and a short form of the Barratt Impulsiveness Scale. International Journal of Neuroscience, 117(3), 359-368. https://doi. org/10.1080/00207450600588881
Thomas, J., Al-Menhali, S., \& Humeidan, M. (2016). Compulsive buying and depressive symptoms among female citizens of the United Arab Emirates. Psychiatry Research, 237, 357-360. https://doi. org/10.1016/j.psychres.2016.01.042

Unger, A., Lyu, H., \& Zimbardo, P. G. (2018). How Compulsive Buying Is Influenced by Time Perspective-Cross-Cultural Evidence from Germany, Ukraine, and China. International Journal of Mental Health and Addiction, 1-20. https://doi. org/10.1007/s11469-018-9942-4

Villardefrancos, E., \& Otero-López, J. M. (2016). Compulsive buying in university students: its prevalence and relationships with materialism, psychological distress symptoms, and subjective wellbeing. Comprehensive Psychiatry, 65, 128-135. https://doi.org/10.1016/j. comppsych.2015.11.007

Williams, A. D. \& Grisham, J. R. (2012). Impulsivity, Emotion Regulation, and Mindful Attentional Focus in Compulsive Buying. Cognitive Therapy Research, 36, 451-457. https://doi.org/10.1007/s10608-011-9384-9

Zhang, C., Brook, J. S., Leukefeld, C. G., \& Brook, D. W. (2016). Associations between compulsive buying and substance dependence/abuse, major depressive episodes, and generalized anxiety disorder among men and women. Journal of Addictive Diseases, 35(4), 298-304. https:// doi.org/10.1080/10550887.2016.1177809

Zhang, C., Brook, J. S., Leukefeld, C. G., De La Rosa, M., \& Brook, D. W. (2017). Compulsive buying and quality of life: An estimate of the monetary cost of compulsive buying among adults in early midlife. Psychiatry Research, 252, 208-214. 